A Fast Visible Camera

Divertor-Imaging Diagnostic on DIII-D

A.L. Roquemore, R. Maingi, C.J. Lasnier, N. Nishino, T.E. Evans, M.E. Fenstermacher, A. Nagy

June 21, 2007

SOFE

Albuquerque, NM, United States

June 17, 2007 through June 22, 2007 
This document was prepared as an account of work sponsored by an agency of the United States Government. Neither the United States Government nor the University of California nor any of their employees, makes any warranty, express or implied, or assumes any legal liability or responsibility for the accuracy, completeness, or usefulness of any information, apparatus, product, or process disclosed, or represents that its use would not infringe privately owned rights. Reference herein to any specific commercial product, process, or service by trade name, trademark, manufacturer, or otherwise, does not necessarily constitute or imply its endorsement, recommendation, or favoring by the United States Government or the University of California. The views and opinions of authors expressed herein do not necessarily state or reflect those of the United States Government or the University of California, and shall not be used for advertising or product endorsement purposes. 


\title{
A Fast visible camera Divertor-imaging diagnostic on DIII-D
}

\author{
A.L. Roquemore ${ }^{1}$, R. Maingi ${ }^{2}$, C.J. Lasnier ${ }^{3}$, N. Nishino ${ }^{4}$ T.E. Evans ${ }^{5}$, \\ M.E. Fenstermacher ${ }^{3}$, A. Nagy ${ }^{1}$, \\ (1) Princeton Plasma Physics Laboratory, Princeton, NJ 08543, USA \\ (2) ' Oak Ridge National Laboratory, Oak Ridge, TN 37831, USA \\ (3) Lawrence Livermore National Laboratory, Livermore, Ca 94550, USA \\ (4) ${ }^{4}$ Hiroshima University, Higashi-Hiroshima, 739-8527 Japan \\ (5) General Atomics San Diego Ca 94551-9090, USA
}

\begin{abstract}
In recent campaigns, the Photron Ultima SE fast framing camera has proven to be a powerful diagnostic when applied to imaging divertor phenomena on the National Spherical Torus Experiment (NSTX). Active areas of NSTX divertor research addressed with the fast camera include identification of types of EDGE Localized Modes (ELMs)[1], dust migration, impurity behavior and a number of phenomena related to turbulence. To compare such edge and divertor phenomena in low and high aspect ratio plasmas, a multi-institutional collaboration was developed for fast visible imaging on NSTX and DIII-D. More specifically, the collaboration was proposed to compare the NSTX small type V ELM regime [2] and the residual ELMs observed during Type I ELM suppression with external magnetic perturbations on DIII-D[3]. As part of the collaboration effort, the Photron camera was installed recently on DIII-D with a tangential view similar to the view implemented on NSTX, enabling a direct comparison between the two machines. The rapid implementation was facilitated by utilization of the existing optics that coupled the visible spectral output from the divertor vacuum ultraviolet UVTV system, which has a view similar to the view developed for the divertor tangential TV camera [4]. A remote controlled filter wheel was implemented, as was the radiation shield required for the DIII-D installation. The installation and initial operation of the camera are described in this paper, and the first images from the DIII-D divertor are presented.
\end{abstract}

\section{Introduction}

Fast frame rate cameras that can be continuously operated during the length of a discharge provide both insight and quantitative information about fast events in laboratory plasmas. Cameras in the range of 1000 frames per second have been routinely used as a plasma monitor for many years. Ultra fast framing rates of greater than $100 \mathrm{kHz}$ are now routinely used to characterize turbulence. Intermediate framing rates of several tens of $\mathrm{kHz}$ are required to record events such as (ELMs). ELMs are beneficial for high performance operations in that they tend to flush excess fuel as well as impurities from the plasma edge. The down side of ELMs is that they can transiently apply a large amount of energy to plasma facing components resulting in enhanced surface erosion. ELM mitigation studies are an active area of research on present day machines with the goal of being able to extrapolate results to next step devices such as ITER. On NSTX, three types of ELMs were initially observed. These are the so-called Type I, Type II and Type III ELMs $[5,6]$. Recently the first visible images of a new type of small edge localized event were successfully recorded and reported in Ref [1], using a Photron Ultima $\mathrm{SE}$ camera. Further analysis of this event led to the designation of a new class of ELMs, referred to as Type $\mathrm{V}$ ELMs [2]. This small ELM shows promise as a new operating regime for future fusion devices in that the benefits of ELMs are retained while the erosion of the plasma facing components is minimized. The desire to determine the universality of the new ELMs, and indeed to study and compare the characteristics between all types of ELMs inspired the idea of applying the Photron camera to another fusion device to study the similarities and differences of the different ELM types. DIII-D was the most favorable tokamak in that it is a large aspect ratio machine with a shape most like ITER and has also recorded Type I through Type IV ELMS. A multinational collaboration to study ELM similarities between DIII-D and NSTX was established between five institutions, PPPL, Hiroshima University, ORNL, GA and LLNL. After two years of operation viewing the NSTX divertor, the camera system was moved to DIII-D to specifically record ELM events. A view similar to the NSTX tangential divertor view was found for the DIII-D divertor. This paper details the installation and presents example data obtained for comparison of the ELM characteristics found on each machine.

\section{Divertor Views}

In order to make comparisons between the two machines, the viewing geometry of each must be similar. ELMs are most interesting in the way they interact with the divertor so that a tangential view of the lower divertor region is expected to be most informative. Fig 1a shows the view of the NSTX divertor and Fig. 1b. shows the similar view for DIII-D. In each view the lower edge of the center column is visible as is a view across the floor of the divertor. The 
far side of the divertor floor is also visible showing a portion of the outer strike point. Having both strike points visible is important in determining the origin and direction of propagation of ELMS.

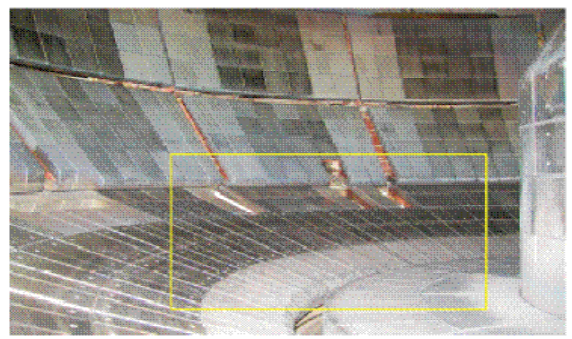

Fig 1a NSTX divertor view

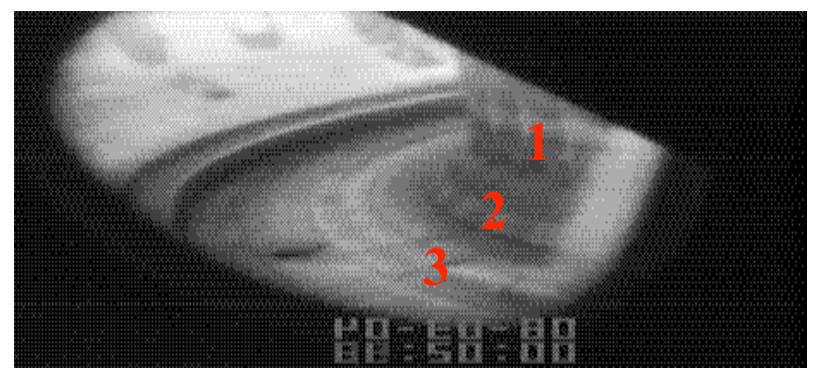

Fig 1b. DIII-D lower divertor view. The center column (1) is connected to the horizontal divertor target( 3 ) by a set of tiles (2) angled at $45^{\circ}$.

\section{Camera/Optics}

The Photron Ultima SE[7] fast framing camera was initially fielded on NSTX as part of collaboration between Hiroshima University and PPPL with the specific purpose of studying divertor phenomena on NSTX. The Photron camera is based on a CMOS sensor technology and has eight bit resolution. At framing rates up to 4500 frames per second (fps), the full sensor array of $256 \mathrm{X} 256$ pixels are utilized. As the framing rate is increased, the pixel set is progressively reduced so that at the fastest rate of $40500 \mathrm{fps}$, the usable array is $64 \mathrm{X} 64$ pixels. This camera has proven to be very robust in the harsh NSTX environment. It has proven to be completely immune to magnetic fields of up to $2.5 \mathrm{kG}$ that it has been subjected to on NSTX which is much greater than the $.5 \mathrm{kG}$ expected for the location on DIII-D. The camera is also moderately resistant to neutron/gamma fluxes as discussed in section IV. The camera and associated optics from NSTX were ideally suited to be mated to the imaging optics on DIII-D making the camera transfer particularly easy.

\section{A. NSTX system}

A description of the camera and optics system applied to the NSTX divertor is discussed in detail in reference [8] and is only briefly described here. The NSTX lower divertor is viewed through a reentrant window at the vertical location of the divertor X-point. The window is on the end of a $50 \mathrm{~cm}$ long and $7 \mathrm{~cm}$ diameter tube. The camera will not physically fit inside the narrow tube so the divertor image is carried from the divertor view port to the camera by an IG-154 fiber optic bundle $2.7 \mathrm{~m}$ long and manufactured by Schott [9]. A filter wheel is placed between the camera lens and the relay lens of the bundle.

\section{B. DIII-D imaging optics}

On DIII-D, an existing vacuum ultraviolet imaging system views the lower divertor and is described in detail in reference [10]. This system utilizes a sophisticated combination of reflective and refractive optics to image the entire divertor view onto a phosphor screen that is $\sim 2 \mathrm{~cm}$ in diameter. The components in the system were chosen to maximize the efficiency in the UV range of wavelengths. This system was adapted to transmit visible light by replacing the $\mathrm{Mg}$ F2 lens with a BK7 lens, removing the imaging phosphor and removing the UV filters in the optical path. The quoted specifications of the system give a magnification $=.023$ and a field of view of $26^{\circ}$. The $f$ number of 8.9 is given for UV wavelengths. Much larger throughput was obtained for the visible range though an attempt to determine the overall $f$ number of the system was not made.

The Photron camera could have been used to directly view the image plane of these existing optics by placing it on the same location as the original CID camera used in the UV application. However, the camera had to be embedded in a radiation shield described in section IV. This again required that the image transfer be made via the same 2.7 meter long imaging bundle used on NSTX. This bundle was outside of the radiation shield and is susceptible to browning in the high flux neutrons from DIII-D. The original bundle became unusable after $\sim 200$ shots and was replaced with a new bundle for the last third of the experiment. The object lens had a focal length of $25 \mathrm{~mm}$ with a $20 \mathrm{~cm}$ c-mount extender inserted between the lens and fiber bundle in order to focus on the $2 \mathrm{~cm}$ high image. The relay lens was an identical match to the camera lens with each focused to infinity so that near parallel light passes between them. This is the ideal location to insert a narrow band filter if required since narrow band filters suffer a cutoff in transmission at angles of incidence of only a few degrees.

\section{Filter Wheel}

Various characteristics of plasmas are best identified with the help of interference filters. For instance, recycling of deuterium from the divertor surfaces is best determined 
by viewing the $\mathrm{H}$-alpha light. The strongest signal from ELMs is also obtained with $\mathrm{H}$-alpha, For machines such as NSTX and DIII-D with carbon plasma facing components, the accumulation of carbon impurities eroded from the divertor surface is best seen with CII and CIII light. In order to cover different operating conditions, several different filters were required. For this application, a $30 \mathrm{~cm}$ diameter filter wheel was installed as an integral part of the radiation shield and had apertures to accommodate five 2" diameter filters. The filters were a narrow band $\mathrm{H}$-alpha, CI,C II,C III, near IR, and HeII. The filter wheel motion is provided for by a Velmex rotary table B4800TS (5") and is remotely controlled through optically coupled RS232 ports.

\section{Radiation Shield}

The Photron camera successfully survived two campaigns in the neutron/gamma radiation fields of NSTX without radiation shielding. By the end of the second campaign, however, an increase in noise attributed to radiation damage was observed and the CMOS sensor was replaced. A neutron yield of $10^{14} \mathrm{n} / \mathrm{sec}$ is typically produced on NSTX with all three neutral beam sources operating at $80 \mathrm{keV}$. On DIII-D, the typical neutron yield is $\sim 100$ times higher with the capability of $5 \mathrm{X}$ higher yields which could easily cause problems in the camera sensor and signal processing electronics. On DIII-D, the location of the camera was at a major radius of 1.8 times greater than on NSTX, reducing the flux by a factor of three. An additional factor of $\sim 50$ is required to further reduce the flux to near the NSTX values. This was accomplished by constructing a two component shield around the camera with the outer layer made of borated $(\sim 5 \%)$ polyethylene and an internal housing of $2-4$ " of lead around the camera. The high hydrogen content of the polyethylene thermalizes the fast neutrons. The boron admixture captures the thermalized neutrons due to its high cross-section for neutron capture at energies of less than $100 \mathrm{ev}$. Neutron attenuation measurements were made at PPPL in the neutron calibration facility using a $20 \mathrm{Ci} \mathrm{PuBe}$ source. The attenuation curves for fast incident neutrons using a moderated $3 \mathrm{He}$ detector are shown in Fig. 2 and were obtained with and without an inner wall of lead bricks. As can be seen, the lead had little effect on the neutron flux reaching the detector.

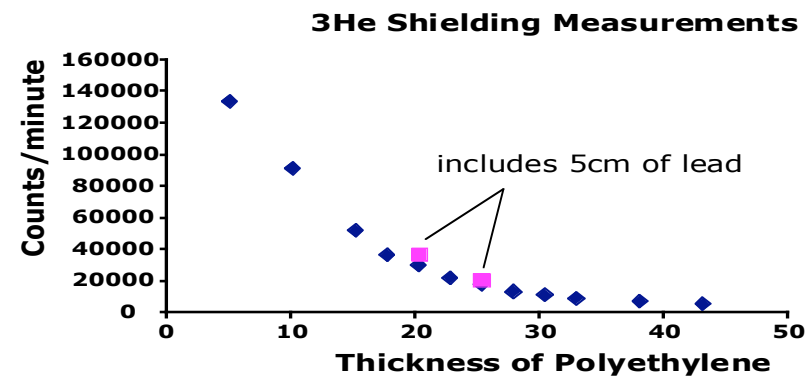

Fig 2. Neutron attenuation measurements

In order to achieve a factor of $\sim 50$ in attenuation, it was determined that a thickness of $35 \mathrm{~cm}$ of polyethylene was required, at least on the end closest to the machine and 25 $\mathrm{cm}$ for the rest of the sides and top.

The gamma flux is generally considered to have the more lethal impact on electronic components. Gamma rays are only slightly attenuated by the polyethylene so that a layer of lead is used to attenuate this component. The majority of incident gammas are produced by $n-\gamma$ reactions in the surrounding structures. A second source of gammas is produced in the borated polyethylene portion of the shield. Neutron capture by hydrogen produces a $2.2 \mathrm{MeV}$ gamma while neutron capture in boron produces a $.48 \mathrm{MeV}$ gamma [11] implying that a borated polyethylene shield alone may actually increase the incident gamma radiation to the camera. The lead shield must be inside the polyethylene housing directly next to the camera. The lead surround was constructed around a cavity just large enough to house the camera body and lenses as shown in Fig. 3.

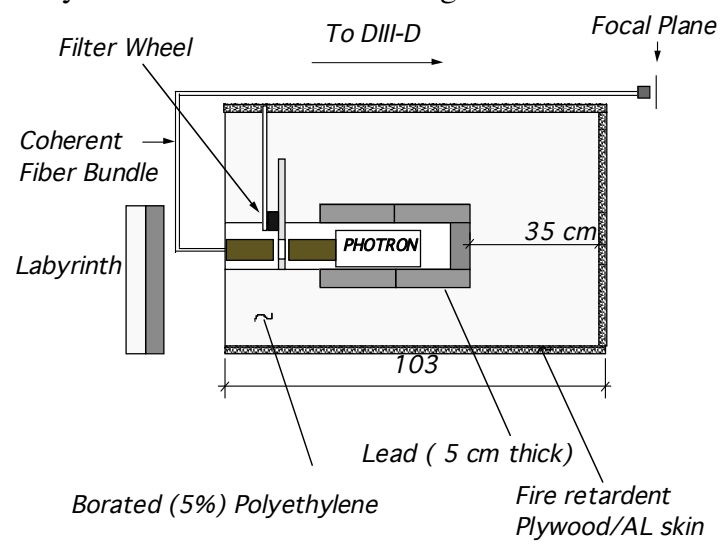

Fig3. Radiation shield for the Photron Camera

The opening in the shield faced away from the machine so the camera was only exposed to scattered radiation. A labyrinth was place in front of the opening to further reduce the incident flux. The signal processing electronics is connected to the camera head via a $15 \mathrm{~m}$ cable. The electronics were placed approximately $12 \mathrm{~m}$ from the machine center and received no evident damage.

The total weight of the final enclosure was $\sim 600$ $\mathrm{kg}$. This included the weight of a fire retardant plywood and aluminum skin place around the shield for fire protection.

DIII-D has the capability of operating at up to $5 \times 10^{16} \mathrm{n} / \mathrm{s}$ which is well beyond the predicted capabilities of the shield. On days when elevated fluxes were expected the camera was removed from the test cell.

Data were obtained for a total of $\sim 500$ DIII-D discharges producing well over the total yearly production of neutrons for NSTX and no degradation of the camera sensor has been observed. 


\section{ELM Data}

An example of the time evolution of a large ELM imaged at 13,500 frames per second with the camera view shown in Fig. 1b (albeit flipped horizontally) is shown in Figure 4. Panel (a) represents the unperturbed (unfiltered) light pattern, with both the inner strike point and outer strike point in view of the camera. The impact of the ELM on the divertor light is seen in panel (b), i.e. both the inner and outer divertor light increased substantially. Panel (c) shows an even brighter light pattern in the next camera frame, and this enhancement occurs at the unperturbed equilibrium strike points. The recycling zone on the inner divertor leg gradually moved to smaller major radius, i.e. up the $45^{\circ}$ tiles, as shown in panel (d). During relaxation of the plasma equilibrium following the ELM, the inner divertor light pattern appeared to split (e), with a less intense band between two more intense bands. The light pattern eventually returned to the unperturbed equilibrium (f), i.e. with the inner strike point near the corner of the $45^{\circ}$ tiles and the horizontal divertor tiles.

This behavior of the recycling zone at the inner strike point is qualitatively consistent with the concept of an ELM as either a peeling mode or a ballooning mode [12]. In the peeling mode scenario, some plasma current near the X-point is expected to be "peeled off' during the ELM. This would temporarily increase the height of the $\mathrm{X}$-point above the divertor floor, which would cause the inner (outer) strike point to shift to smaller (larger) major radius. The recycling zone would follow the inner strike point, and then relax back to the pre-ELM equilibrium as the current density in the X-point region relaxed to preELM levels. A detailed comparison of the evolution of the light pattern with expected strike point shifts is beyond the scope of this paper and left for a future study.

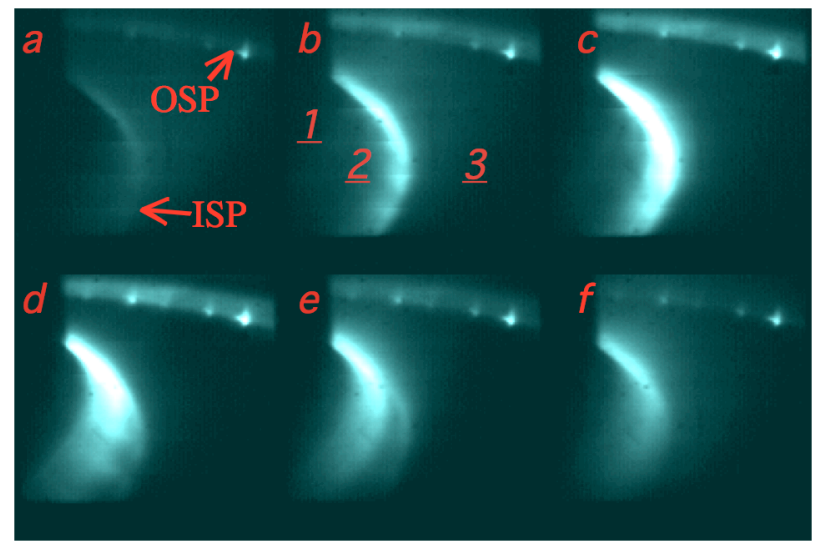

Fig 4. Time evolution of a large, Type I ELM as imaged by the fast camera from discharge $\# 122517$ at 13,500 frames per second. The center column (1), 450 inboard tiles (2), and horizontal divertor target (3) as shown in Fig. $1 \mathrm{~b}$ are labeled in panel $\mathrm{b}$. The inner strike point (ISP) and outer strike point (OSP) are labeled in panel a. The time of the panels relative to panel a are: $\mathrm{b}-74 \mu \mathrm{sec}, \mathrm{c}-$ $148 \mu \mathrm{sec}, \mathrm{d}-741 \mu \mathrm{sec}, \mathrm{e}-1556 \mu \mathrm{sec}$, and $\mathrm{f}-2296 \mu \mathrm{sec}$. The periodic bright zones along the outer strike point correspond to tile edges.

\section{Summary}

The Photron camera was successfully operated on DIII-D with the aid of a radiation shield. Two types of ELMs have been characterized between the machines and the data is being analyzed. Type V ELMS have not yet been identified on DIII-D. Work is underway to compare images of Type I and Type III ELMS that are common to both machines.

\section{References}

[1]N. Nishino, et. al., 2002 J. Plasma Fusion Res. 78 1278.

[2]R. Maingi, et. al., 2005 Nuclear Fusion 45264.

[3]T. E. Evans, et. al., 2004 Physical Review Letters 92 article \#235003.

[4]M.E. Fenstermacher, et. al., J. Nucl. Mater. 241-3 (1997) 666

[5] Maingi J. Nucl. Mater. 337-339 (2005) 727

[ 6]N. Nishino et al., Proceedings of the Twenty-first Symposium on Fusion Engineering, Knoxville, Tennessee, Sept. 2005, IEEE.

[7]Photron Ltd., Fujimi-cho 1-1-8, Chiyoda-ku, Tokyo

[8]A. L. Roquemore, et. al., 2004 Review of Scientific Instruments $\mathbf{7 5} 4190$.

[9] Schott North America, 122 Charlton Street Southbridge, MA 01550102-0071

[10]D. G. Nilson, et. al., 1999 Review of Scientific Instruments $\mathbf{7 0} 738$.

[11] Glenn F. Knoll, Radiation Detection and Measurement, John Wiley and Sons, New York (1979)

[12] Snyder Phys Plasma 9 (2002) 2037

This work was performed under the auspices of the U.S. Department of Energy by University of California, Lawrence Livermore National Laboratory under Contract W-7405-Eng-48. 